University of Warwick institutional repository: http://go.warwick.ac.uk/wrap This paper is made available online in accordance with publisher policies. Please scroll down to view the document itself. Please refer to the repository record for this item and our policy information available from the repository home page for further information.

To see the final version of this paper please visit the publisher's website. Access to the published version may require a subscription.

Author(s): JÉRÔME BUZZI and OMRI SARIG

Article Title: Uniqueness of equilibrium measures for countable Markov shifts and multidimensional piecewise expanding maps

Year of publication: 2003

Link to published

version: http://dx.doi.org/10.1017/S0143385703000087

Publisher statement: None 


\title{
Uniqueness of equilibrium measures for countable Markov shifts and multidimensional piecewise expanding maps
}

\author{
JÉRÔME BUZZI $\dagger$ and OMRI SARIG $\ddagger$ \\ † CMAT, U.M.R. 7640 du C.N.R.S., Ecole Polytechnique, 91128 Palaiseau Cedex, France \\ (e-mail: buzzi@math.polytechnique.fr) \\ $\$$ Mathematics Institute, University of Warwick, Coventry CV4 7AL, UK \\ (e-mail: sarig@maths.warwick.ac.uk)
}

(Received 12 July 2002 and accepted in revised form 23 October 2002)

\begin{abstract}
We prove that potentials with summable variations on topologically transitive countable Markov shifts have at most one equilibrium measure. We apply this to multidimensional piecewise expanding maps using their Markov diagrams.
\end{abstract}

\section{Introduction}

Let $S$ be a countable set and $A=\left(t_{i j}\right)_{S \times S}$ a matrix of zeroes and ones. The (one-sided) topological Markov shift with set of states $S$ and transition matrix $A$ is

$$
\Sigma=\Sigma_{A}^{+}:=\left\{\left(x_{0}, x_{1}, \ldots\right) \in S^{\mathbb{N} \cup\{0\}}: t_{x_{i} x_{i+1}}=1 \text { for all } i\right\}
$$

together with the action of the left shift $\sigma: \Sigma \rightarrow \Sigma$. The topology on $\Sigma$ is assumed to be the relative product topology inside $S^{\mathbb{N} \cup\{0\}}, S$ being discrete. A shift invariant probability measure $\mu$ is called a maximal measure if $h_{\mu}(\sigma)$ is maximal and an equilibrium measure for $\phi: \Sigma \rightarrow \mathbb{R}$ if $h_{\mu}(\sigma)+\int \phi d \mu$ is well-defined and maximal.

Parry proved in [19] that if $S$ is finite and $\sigma$ is topologically transitive, then there exists exactly one maximal measure and that this measure is the Markov measure with initial distribution $\left(p_{i}\right)$ and transition matrix $\left(p_{i j}\right)$ where $p_{i}=u_{i} v_{i}, p_{i j}=u_{j} t_{i j} / \lambda u_{i}$ and $u=\left(u_{i}\right), v=\left(v_{i}\right)$ and $\lambda>0$ are given by $v A=\lambda v, A u=\lambda u$ and $\langle u, v\rangle=1$.

Ruelle [20] improved on this and showed that if $S$ is finite, $\sigma$ is topologically transitive and $\operatorname{var}_{n}(\phi)=O\left(\theta^{n}\right)$ for some $\theta \in(0,1)$, where

$$
\operatorname{var}_{n}(\phi):=\sup \left\{\phi(x)-\phi(y): x_{i}=y_{i} \text { for } i=0, \ldots, n-1\right\},
$$

then $\phi$ has exactly one equilibrium measure. Furthermore, he showed that if $L_{\phi}$ is the operator $L_{\phi} f=\sum_{\sigma y=x} e^{\phi(y)} f(y)$, then the unique equilibrium measure is given by $h d v$ 
where $h$ and $v$ are a positive continuous function and a Borel probability measure such that $\int h d v=1, L_{\phi} h=\lambda h$ and $L_{\phi}^{*} v=\lambda v$ for $\lambda>0$. The condition $\operatorname{var}_{n}(\phi)=O\left(\theta^{n}\right)$ was relaxed to $\sum \operatorname{var}_{n}(\phi)<\infty$ by Walters [27].

If $S$ is infinite there may be no maximal measure, but if it exists it must be unique as long as $\sigma$ is topologically transitive (Gurevich $[\mathbf{1 0}, \mathbf{1 1}]$ ). Indeed, Gurevich showed that in this case there exists a maximal measure if and only if $A$ is $R$-recurrent and $R$-positive and also in this case the unique maximal measure can be determined as in the case $|S|<\infty$ (the $R$-positivity and $R$-recurrence conditions are necessary and sufficient conditions for the existence of $u$ and $v$ ). The same techniques yield the uniqueness of equilibrium measures of potentials of the form $\phi(x)=\phi\left(x_{0}, \ldots, x_{N}\right)$ (Gurevich and Savchenko [12]).

Our aim in this paper is to give a Ruelle-type generalization of these results for $\phi$ which depend on an infinite number of coordinates such that $\sum_{n \geqslant 2} \operatorname{var}_{n}(\phi)<\infty$. We then apply this generalization to certain (non-Markovian) multidimensional piecewise expanding maps.

The Gurevich pressure of $\phi$ is $P_{G}(\phi):=\lim _{n \rightarrow \infty}(1 / n) \log \sum_{\sigma^{n} x=x} e^{\phi_{n}(x)} 1_{[a]}(x)$ where $a \in S$ is fixed, $[a]:=\left\{x \in \Sigma: x_{0}=a\right\}$ and $\phi_{n}:=\sum_{i=0}^{n-1} \phi \circ T^{i}$. Let $\mathcal{P}_{\sigma}(\Sigma)$ denote the collection of $\sigma$-invariant Borel probability measures on $\Sigma$. The metric pressure (or just pressure) of $\mu \in \mathcal{P}_{\sigma}(\Sigma)$ is:

$$
P_{\mu}(\phi)=P_{\mu}(\phi, \sigma):=h_{\mu}(\sigma)+\int \phi d \mu .
$$

Note that this is not always well-defined ( $\phi$ might not be integrable, or it might happen that $h_{\mu}(\sigma)=+\infty$ and $\left.\int \phi d \mu=-\infty\right)$. One of us showed [22, 23] that if $\sigma$ is topologically mixing and $\sup \phi<\infty$, then

$$
P_{G}(\phi)=\sup \left\{P_{\mu}(\phi): \mu \in \mathcal{P}_{\sigma}(\Sigma), P_{\mu}(\phi) \text { is well-defined }\right\} .
$$

The condition $\sup \phi<\infty$ guarantees that $\int \phi d \mu$ is well defined (though possibly infinite), so the 'well defined' condition reduces to a preclusion of the $\mu$ for which $h_{\mu}(\sigma)=\infty$ and $\int \phi d \mu=-\infty$. $\dagger$ We prove the following.

THEOREM 1.1. Let $(\Sigma, \sigma)$ be a topologically transitive countable Markov shift and suppose $\phi: \Sigma \rightarrow \mathbb{R}$ satisfies $\sup \phi<\infty, P_{G}(\phi)<\infty$ and $\sum_{n \geqslant 2} \operatorname{var}_{n}(\phi)<\infty$. There exists at most one invariant probability measure $\mu$ such that $h_{\mu}(\sigma)+\int \phi d \mu$ is well defined and maximal.

THEOREM 1.2. Under the assumptions of the previous theorem, if $\mu$ exists, then there exist a positive continuous function $h$ and a Borel measure $v$ finite on each $[a], a \in S$ and with full support such that for some $\lambda>0, L_{\phi} h=\lambda h, L_{\phi}^{*} v=\lambda \nu$ and $\int h d v=1$. Moreover, $d \mu=h d v$.

These two theorems show that for $\phi$ with summable variations and finite Gurevich pressure, if $\phi$ has an equilibrium measure then it is positive recurrent in the terminology of $[\mathbf{2 3}, \mathbf{2 4}]$. The opposite is not true, because it might happen that $d \mu=h d v$ where

$\dagger$ Measures $\mu$ with $\int \phi d \mu=-\infty$ cannot contribute to the supremum (which is always larger than $-\infty$ because of the existence of invariant measures supported on periodic points). Therefore, the condition of $h_{\mu}+\int \phi$ being well defined can be replaced by the condition $-\int \phi<\infty$. 
$L_{\phi}^{*} v=\lambda \nu, L_{\phi} h=\lambda h$ and $\int f d v=1$, satisfies $h_{\mu}(\sigma)=\infty$ and $\int \phi d \mu=-\infty$ (in which case $h_{\mu}(\sigma)+\int \phi d \mu$ is meaningless). Nevertheless, even in these situations, the measure $d \mu=h d v$ can still be interpreted as some kind of weak equilibrium measure. We refer the reader to $[\mathbf{2 3}, \mathbf{2 4}]$ for details.

Finally, we remark that if the equilibrium measure of $\phi$ is a Gibbs measure (see [2]), then its uniqueness can be deduced from the uniqueness of Gibbs measures, as in [2]. Unfortunately, if $|S|=\infty$ this never happens, unless $A$ satisfies the BIP property (see [18] and [26] for statements and definitions), so the general case cannot be treated this way.

Theorem 1.1 can be applied to non-Markov, multidimensional piecewise expanding maps by using the connected Markov diagram introduced in [5]. To state our result we need some definitions.

A piecewise expanding map $(X, P, T)$ is a locally connected compact metric space $X$ together with a 'partition' $P$ which is simply a finite collection of non-empty, pairwise disjoint open subsets of $X$ with dense union and a map $T: \bigcup_{A \in P} A \rightarrow X$ such that for each $A \in P$, each restriction $\left.T\right|_{A}$ can be extended to an expanding homeomorphism between a neighborhood of $\bar{A}$ and one of $\overline{T A}$.

The boundary of such a system is $\partial P:=\bigcup_{A \in P} \partial A$.

$P_{0}^{n-1}$ denotes the collection of $n$-cylinders, i.e. the non-empty intersections $A_{0} \cap$ $T^{-1} A_{1} \cap \cdots \cap T^{-n+1} A_{n-1}$ where $A_{i}$ are elements of $P$.

A piecewise Hölder-continuous potential is $\phi: X \rightarrow \mathbb{R}$ such that the restriction of $\phi$ to any element of $P$ is Hölder-continuous, i.e. for all $x, y$ in the same element of $P$,

$$
|\phi(x)-\phi(y)| \leqslant K d(x, y)^{\alpha}
$$

for some $\alpha>0, K<\infty$ (the values of $\phi$ on $\partial P$ are irrelevant for our purposes).

The pressure of a subset $S \subseteq X$ (not necessarily invariant) is

$$
P_{\text {top }}(\phi, S, T):=\limsup _{n \rightarrow \infty} \frac{1}{n} \log \sum_{A \in P_{0}^{n-1} \mid S \cap \bar{A} \neq \varnothing} \sup _{x \in A} \exp \phi_{n}(x)
$$

where $\phi_{n}(x):=\phi(x)+\phi(T x)+\cdots+\phi\left(T^{n-1} x\right)$.

The topological pressure of $T$ is $P_{\mathrm{top}}(\phi, T):=P_{\mathrm{top}}(X, T)$.

An equilibrium measure is, as in the case of Markov shifts, an invariant probability measure $\mu$ for which the metric pressure $P_{\mu}(\phi, T)$ is equal to the topological pressure $P_{\text {top }}(\phi, T)$.

THEOREM 1.3. Let $(X, P, T)$ be a piecewise expanding map with a piecewise Höldercontinuous potential $\phi$. Assume that

$$
P_{\text {top }}(\phi, \partial P, T)<P_{\text {top }}(\phi, T) .
$$

Then:

(i) $\quad P_{\text {top }}(\phi, T)=\sup _{\mu \in \mathcal{P}_{T}(X)} P_{\mu}(\phi, T)$ and this supremum is realized by at least one measure;

(ii) there exist at most finitely many ergodic equilibrium measures; and

(iii) if, additionally, $T$ is strongly topologically transitive in the sense that for all nonempty open sets $U, \bigcup_{k \geqslant 0} T^{k}(U) \supseteq T(X)$, then there is a unique equilibrium measure. 
Example. Recall that a multidimensional $\beta$-transformation is a map $T:[0,1)^{d} \rightarrow[0,1)^{d}$, $d \geqslant 1$ of the form $T(x)=\{B(x)\}$ where $B: \mathbb{R}^{d} \rightarrow \mathbb{R}^{d}$ is an expanding affine map and $\{y\}$ is the unique vector in $[0,1)^{d}$ equal to $y \bmod \mathbb{Z}^{d}$. $T$ is obviously a piecewise expanding map on $[0,1]^{d}$, the partition $P$ being the collection of maximum connected open sets of continuity.

COROLlARY 1.4. A multidimensional $\beta$-transformation $T$ admits finitely many ergodic equilibrium measures with respect to any non-negative Hölder-continuous potential $\phi$ with $\sup \phi<\lambda_{d}:=\log \min \left\{\left\|B_{\operatorname{lin} .} v\right\|:\|v\|=1\right\}, B_{\text {lin }}$ being the linear part of the affine map $B$.

Moreover if $\lambda_{d}>\log (1+\sqrt{d})$ then the equilibrium measure is unique.

To see that the corollary follows from the theorem observe that, $\partial P$ being included in a finite union of hyper planes, Proposition 4 of [4] gives

$$
P_{\text {top }}(\phi, \partial P, T) \leqslant P_{\text {top }}(0, \partial P, T)+\sup \phi \leqslant \lambda_{1}+\cdots+\lambda_{d-1}+\sup \phi
$$

and

$$
P_{\text {top }}(\phi, T) \geqslant \lambda_{1}+\cdots+\lambda_{d}
$$

with $\lambda_{1} \geqslant \cdots \geqslant \lambda_{d}$ the logarithms of the moduli of the eigenvalues of $B_{\text {lin. }}$. It follows that

$$
P_{\text {top }}(\phi, \partial P, T) \leqslant P_{\text {top }}(\phi, T)-\left(\lambda_{d}-\sup \phi\right)<P_{\text {top }}(\phi, T)
$$

so that one can indeed apply Theorem 1.3 to get the finiteness.

The uniqueness follows from Proposition 1 of [4], according to which the lower-bound on the expansion of the statement of the Corollary implies strong topological mixing.

Theorem 1.3 generalizes a result of [6] which required the existence of a conformal measure with full support-which had only been proved under additional assumptions (especially covering, a strong form of mixing) by [8]. Indeed, it is easy to build examples where the equilibrium measure is supported by a Cantor set and this shows that [6] cannot apply, since in this case the support would contain a non-empty open set (more precisely, the support would be equal to an open set modulo a set with zero conformal measure).

The core of the proof of this statement is the isomorphism theorem, Theorem 3.2, identifying $T$ and the Markov shift defined by its so-called connected Markov extension with respect to all measures with metric pressure sufficiently close to $P_{\text {top }}(\phi, T)$.

\section{Equilibrium measures on the Markov shift}

This section contains the proofs of Theorems 1.1 and 1.2. We use the cylinder notation $\left[a_{0}, \ldots, a_{n-1}\right]:=\left\{x \in \Sigma: x_{i} \in\left[a_{i}\right]\right\}$ and set $\alpha:=\{[a]: a \in S\}$.

LEMMA 2.1. Suppose $(\Sigma, \sigma)$ is topologically transitive and $\phi: \Sigma \rightarrow \mathbb{R}$ is such that $\sup _{n \geqslant 1} \operatorname{var}_{n+1}\left(\phi_{n}\right)<\infty$. Let $v$ be a conservative $\sigma$-finite measure which is finite and positive on some partition set. If $L_{\phi}^{*} v=\lambda \nu$ for some $\lambda>0$, then $v$ is ergodic.

Proof. We indicate the proof, which is well known (see [1, Ch. 4]). If $v$ is such a measure, then its transfer operator is $\lambda^{-1} L_{\phi}$ and it is easy to see that if $C:=\exp \sup _{n \geqslant 1} \operatorname{var}_{n+1}\left(\phi_{n}\right)$, then for every $[\underline{a}]=\left[a_{0}, \ldots, a_{n-1}\right] \in \alpha_{0}^{n-1},[\underline{b}] \in \alpha_{0}^{m-1}$ with $[\underline{b}] \subseteq\left[a_{n-1}\right]$ and $x \in[\underline{a}]$,

$$
\frac{1}{C} \lambda^{-(n-1)} e^{\phi_{n-1}(x)} v[\underline{b}] \leqslant v\left([\underline{a}] \cap \sigma^{-(n-1)}[\underline{b}]\right) \leqslant C \lambda^{-(n-1)} e^{\phi_{n-1}(x)} v[\underline{b}] .
$$


Summing over $[\underline{b}] \in \alpha_{0}^{m-1} \cap\left[a_{n-1}\right]$ gives

$$
\frac{1}{C} \lambda^{-(n-1)} e^{\phi_{n-1}(x)} \nu\left[a_{n-1}\right] \leqslant \nu[\underline{a}] \leqslant C \lambda^{-(n-1)} e^{\phi_{n-1}(x)} \nu\left[a_{n-1}\right] .
$$

This proves that every cylinder which ends with a state $c$ such that $v[c]>0$ has positive $v$-measure. By transitivity, we can always find a cylinder contained in $[\underline{a}]$ with this property. Therefore, $v$ is finite positive on all cylinders (although the total mass may be infinite). This argument also shows that

$$
\frac{1}{C} \leqslant \frac{\lambda^{-(n-1)} e^{\phi_{n-1}(x)}}{\nu[\underline{a}] / v\left[a_{n-1}\right]} \leqslant C .
$$

Substituting this in the first displayed inequality gives

$\frac{1}{C^{2}} \frac{v[\underline{a}] v[\underline{b}]}{v\left[a_{n-1}\right]} \leqslant v\left([\underline{a}] \cap \sigma^{-(n-1)}[\underline{b}]\right) \leqslant C^{2} \frac{v[\underline{a}] v[\underline{b}]}{v\left[a_{n-1}\right]} \quad$ for all $[\underline{a}] \in \alpha_{0}^{n-1}$ and $[\underline{b}] \subseteq\left[a_{n-1}\right]$.

A monotone class argument shows that for every Borel set $E$,

$$
\nu\left([\underline{a}] \cap \sigma^{-(n-1)} E\right) \geqslant \frac{1}{C^{2}} \nu[\underline{a}] \nu\left(E \cap\left[a_{n-1}\right]\right) / \nu\left[a_{n-1}\right] .
$$

We can now prove ergodicity. Suppose $E$ is an invariant set of positive measure. The previous estimate shows that

$$
\mathbb{E}_{\nu}\left(1_{E} \mid \alpha_{0}^{n-1}\right)(x)=\frac{\nu\left(\left[x_{0}, \ldots, x_{n-1}\right] \cap T^{-(n-1)} E\right)}{\nu\left[x_{0}, \ldots, x_{n-1}\right]} \geqslant \frac{1}{C^{2}} \frac{v\left(E \cap\left[x_{n-1}\right]\right)}{\nu\left[x_{n-1}\right]} .
$$

By conservativity, $x_{n-1}=x_{0}$ for infinitely many $n$. Consequently

$$
\liminf _{n \rightarrow \infty} \mathbb{E}_{\nu}\left(1_{E} \mid \alpha_{0}^{n-1}\right)(x) \geqslant \frac{1}{C^{2}} \frac{v\left(E \cap\left[x_{0}\right]\right)}{\nu\left[x_{0}\right]} \quad \text { almost everywhere. }
$$

We claim that the right-hand side is positive. This will prove the lemma, since the righthand side is equal (by the Martingale Convergence Theorem) to $1_{E}$ and an indicator of a set can only be positive almost everywhere if $E=\Sigma$ modulo $v$.

By assumption $v(E)>0$, so there exists a state $y_{0}$ such that $v\left(E \cap\left[y_{0}\right]\right)>0$. By transitivity there exist $n$ and $[\underline{a}] \in \alpha_{0}^{n-1}$ such that $[\underline{a}] \subseteq\left[x_{0}\right] \cap \sigma^{-(n-1)}\left[y_{0}\right]$. Then

$$
v\left(E \cap\left[x_{0}\right]\right) \geqslant v\left(\sigma^{-(n-1)} E \cap[\underline{a}]\right) \geqslant \frac{1}{C^{2}} \frac{v[\underline{a}] \nu\left(E \cap\left[y_{0}\right]\right)}{v\left[y_{0}\right]}>0
$$

by the choice of $y_{0}$.

Our next lemma states that it is possible to assume without loss of generality that $(\Sigma, \sigma)$ is topologically mixing.

LEMMA 2.2. If Theorems 1.1 and 1.2 are true for all topologically mixing shifts, they are true for all topologically transitive shifts.

Proof. We use the well-known spectral decomposition (see Remark 7.1.35 in [16]). If $\Sigma$ is topologically transitive, then there exist pairwise disjoint closed sets $\Sigma_{0}, \ldots, \Sigma_{p-1}$ such 
that $\Sigma=\bigcup_{i=0}^{p-1} \Sigma_{i}, \sigma\left(\Sigma_{i}\right)=\Sigma_{(i+1) \bmod p}$, and such that $\left(\Sigma_{i}, \sigma^{p}\right)$ is topologically mixing. Moreover, each $\Sigma_{i}$ is the union of partition sets.

Recoding $\left(\Sigma_{i}, \sigma^{p}\right)$ by the partition into cylinders of length $p$, we may view it as a topologically mixing topological Markov shift.

In order to prove Theorem 1.1 for $\sigma$, it is enough to prove that there exists a unique ergodic invariant $\mu \in \mathcal{P}_{\sigma}(\Sigma)$ with $h_{\mu}(\sigma)+\int \phi d \mu=P_{G}(\phi)$, because if there were more than one invariant equilibrium measure, there would have been more than one ergodic equilibrium measure. Suppose, then, that $\mu$ is an ergodic equilibrium measure.

Note that $\mu\left(\Sigma_{i}\right)=1 / p>0$ for every $i$, because $\Sigma=\bigcup_{k=0}^{p-1} \sigma^{-k}\left(\Sigma_{i}\right)$. Let $\mu_{i}$ be the measure $\mu_{i}(E):=\mu_{i}\left(E \cap \Sigma_{i}\right) / \mu\left(\Sigma_{i}\right)$. Since $\mu=\left(\mu_{0}+\cdots+\mu_{p-1}\right) / p$,

$$
\begin{aligned}
\frac{1}{p} \sum_{i=0}^{p-1} P_{\mu_{i}}\left(\phi_{p}, \sigma^{p}\right) & =P_{\mu}\left(\phi_{p}, \sigma^{p}\right)=p P_{\mu}(\phi, \sigma) \equiv p P_{G}(\phi, \sigma) \\
& =P_{G}\left(\phi_{p}, \sigma^{p}\right) \geqslant \max _{i} P_{G}\left(\phi_{p}\left|\Sigma_{i}, \sigma^{p}\right|_{\Sigma_{i}}\right) .
\end{aligned}
$$

This is possible only if for every $i, \mu_{i}$ is an equilibrium measures of $\phi_{p}$ with respect to $\sigma^{p}: \Sigma_{i} \rightarrow \Sigma_{i}$. This system is topologically mixing, so the $\mu_{i}$ are uniquely determined. These determine $\mu$. This proves the topological transitive version of Theorem 1.1, given its topological mixing version.

Next, note that the topologically mixing version of Theorem 1.2 implies that each $\mu_{i}$ above satisfies $d \mu_{i}=h_{i} d \nu_{i}$, where $h_{i}$ is a continuous function supported and positive on $\Sigma_{i}$ with $L_{\phi}^{p} h_{i}=\lambda_{i}^{p} h_{i}, \lambda>0$ and $\nu_{i}$ is a Borel measure finite and positive on cylinders included in $\Sigma_{i}$ with $\left(L_{\phi}^{p}\right)^{*} v_{i}=\lambda_{i}^{p} v_{i}$. Set

$$
h:=\sum_{i=0}^{p-1} \lambda_{0}^{-i} L_{\phi}^{i} h_{0}, \quad v:=\sum_{i=0}^{p-1} \lambda_{0}^{-i}\left(L_{\phi}^{i}\right)^{*} \nu_{0} \quad \text { and } \quad d m:=\frac{h d v}{\int h d v} .
$$

It is easy to check that $L_{\phi} h=\lambda_{0} h$ and $L_{\phi}^{*} v=\lambda_{0} v$. It follows from this that $m$ is invariant. It is an ergodic measure, since $v$ is ergodic by Lemma 2.1. Our equilibrium measure $\mu$ is also ergodic, because almost all its ergodic components are equilibrium measures and there is only one such measure. Therefore, $\mu$ and $m$ are two ergodic invariant probability measures and by construction $\left.\mu\right|_{\Sigma_{0}}$ and $\left.m\right|_{\Sigma_{0}}$ are proportional. Ergodicity now implies that they are equal. This shows that $\mu$ has the form $h d v$ where $h$ and $v$ are eigenvectors of $L_{\phi}$.

For the remainder of the section, we only treat topologically mixing Markov shifts (the previous lemma states that this is enough).

Definition 1. Let $(\Sigma, \sigma)$ be a topologically mixing countable Markov shift. A function $g: \Sigma \rightarrow \mathbb{R}$ is called a sub $g$-function if $g$ is strictly positive, $P_{G}(\log g)=0$ and for all $x \in \Sigma, \sum_{\sigma y=x} g(y) \leqslant 1$.

We recall the following known results.

LEMMA 2.3. If $(\Sigma, \sigma)$ is topologically mixing, then every $\phi: \Sigma \rightarrow \mathbb{R}$ such that $P_{G}(\phi)<\infty$ and $\sum_{n \geqslant 2} \operatorname{var}_{n}(\phi)<\infty$ is of the form $\log g+\varphi-\varphi \circ \sigma+P_{G}(\phi)$ where $g$ is a sub g-function and $\varphi$ is continuous with $\operatorname{var}_{n}(\varphi) \leqslant \sum_{k \geqslant n+1} \operatorname{var}_{k}(\phi)$ for all $n \geqslant 1$. 
Proof. See Lemma 1 in [25]. We remark that this proof uses the generalized Ruelle's Perron-Frobenius Theorem of $[\mathbf{2 4}, \mathbf{2 5}]$. This is the only place we use this theorem and it would be a significant improvement to have a different argument which does not require it.

LEMmA 2.4. Let $p_{i}, x_{i}(i=1,2,3, \ldots)$ be real numbers such that $p_{i} \geqslant 0, x_{i}>0$ and $\sum p_{i}=1$. If $\sum_{i=1}^{\infty} p_{i} \log x_{i}=\log \left(\sum_{i=1}^{\infty} p_{i} x_{i}\right)$, then all $x_{i}$ with $p_{i} \neq 0$ are equal.

Proof. This is a standard fact.

Proof of Theorem 1.1. By Lemma 2.2 it is enough to treat the topologically mixing case. Assume without loss of generality that $P_{G}(\phi)=0$ (else pass to $\phi-P_{G}(\phi)$ ). By Lemma 2.3 there exists a sub $g$-function and a continuous function $\varphi: \Sigma \rightarrow \mathbb{R}$ with $\operatorname{var}_{1}(\varphi)<\infty$ such that

$$
\phi=\log g+\varphi-\varphi \circ \sigma .
$$

Our strategy of proof is to show that every ergodic equilibrium measure $\mu$ satisfies

$$
L_{\phi}^{*}\left(e^{-\varphi} \mu\right)=e^{-\varphi} \mu .
$$

(Here and throughout an equilibrium measure means an invariant probability measure $\mu$ for which $h_{\mu}(\sigma)+\int \phi d \mu$ is well defined and maximal.)

Once we prove this we can proceed as follows. Assume by way of contradiction that there is more than one invariant equilibrium measure. Every equilibrium measure is a barycenter of the collection of ergodic equilibrium measures, since almost every ergodic component of an equilibrium measure is itself an equilibrium measure. Therefore, if there is more than one equilibrium measure, there must be more than one ergodic equilibrium measure. Let $\mu_{1}$ and $\mu_{2}$ be two different ergodic equilibrium measures and set $\mu:=\frac{1}{2}\left(\mu_{1}+\mu_{2}\right)$. This is a non-ergodic measure which satisfies (2), in contradiction to Lemma $2.1 \dagger$. This contradiction proves the theorem.

Recall that an invariant probability measure $\mu$ is said to satisfy the Rokhlin formula, if

$$
h_{\mu}(\sigma)=-\int \log \frac{d \mu}{d \mu \circ \sigma} d \mu
$$

where $\mu \circ \sigma$ is the $\sigma$-finite measure $(\mu \circ \sigma)(E)=\sum_{A \in \alpha} \mu(\sigma(E \cap A)) \ddagger$. Ledrappier showed in [17] that $\S$

$$
\mathbb{E}_{\mu}\left(f \mid \alpha_{1}^{\infty}\right)(x)=\sum_{\sigma y=\sigma x} \frac{d \mu}{d \mu \circ \sigma}(y) f(y)
$$

and deduced that

$$
I_{\mu}\left(\alpha \mid \alpha_{1}^{\infty}\right):=-\sum_{A \in \alpha} 1_{A} \log \mathbb{E}_{\mu}\left(1_{A} \mid \alpha_{1}^{\infty}\right)=-\log \frac{d \mu}{d \mu \circ \sigma} .
$$

$\dagger$ The conditions of Lemma 2.1 are satisfied: (1) $e^{-\varphi} \mu$ is conservative since it is equivalent to the invariant probability measure $\mu$; (2) $e^{-\varphi} \mu$ is finite and positive on some partition set, since $\mu$ is a probability measure and $\operatorname{var}_{1}(\varphi)<\infty$.

\$ Note that, in general, $(\mu \circ \sigma)(E) \neq \mu(\sigma(E))$.

$\S$ Ledrappier considered the case of a finite alphabet, but this part of his arguments applies to countable alphabets without modifications 
It follows from this that

$$
H\left(\alpha \mid \alpha_{1}^{\infty}\right) \equiv \int I_{\mu}\left(\alpha \mid \alpha_{1}^{\infty}\right) d \mu=-\int \log \frac{d \mu}{d \mu \circ \sigma} d \mu .
$$

If $H_{\mu}(\alpha)$ is finite, then $H\left(\alpha \mid \alpha_{1}^{\infty}\right)=h_{\mu}(\sigma)$. Therefore, Rokhlin's formula holds whenever $H_{\mu}(\alpha)<\infty$.

A key step in the proof of (2) is to show that every ergodic equilibrium measure $\mu$ satisfies the Rokhlin formula, the difficulty being that, in general, $H_{\mu}(\alpha)$ may be infinite so it is not obvious a priori that $H\left(\alpha \mid \alpha_{1}^{\infty}\right)=h_{\mu}(\sigma)$.

Let $\mu$ be an ergodic probability measure for which $h_{\mu}(\sigma)+\int \phi d \mu$ is well defined and equal to zero. Note that $\int \phi d \mu$ must then be finite.

Fix some $[a] \in \alpha$ with positive measure and consider the induced transformation on $[a]$ given by $\bar{\sigma}(x)=\sigma^{\tau(x)}(x)$ where $\tau(x):=1_{[a]}(x) \inf \left\{n \geqslant 1: \sigma^{n}(x) \in[a]\right\}$. The induced transformation preserves the measure $\bar{\mu}(E):=\mu(E \cap[a]) / \mu[a]$ and admits the Markov partition

$$
\beta:=\left\{\left[a, \xi_{1}, \ldots, \xi_{n-1}, a\right]: n \geqslant 1, \xi_{i} \neq a\right\} \backslash\{\varnothing\} .
$$

Define a Bernoulli measure $\bar{\mu}_{B}$ on $[a]$ by $\bar{\mu}_{B}\left(\bigcap_{i=0}^{n-1} \bar{\sigma}^{-i} B_{i}\right)=\prod_{i=0}^{n-1} \bar{\mu}\left(B_{i}\right)$ whenever $B_{i} \in \beta$ and let $\mu_{B}$ be the measure on $\Sigma$ given by

$$
\mu_{B}(E)=\mu[a] \int_{[a]} \sum_{i=0}^{\tau-1} 1_{E} \circ \sigma^{i} d \bar{\mu}_{B}
$$

This is a probability measure, since $\mu_{B}(\Sigma)=\mu[a] \int \tau d \bar{\mu}_{B}=\mu[a] \int \tau d \bar{\mu}=1$ (by the Kac formula). $\mu_{B}$ is $\sigma$-ergodic, since $\bar{\mu}_{B}$ is $\bar{\sigma}$-ergodic and $[a]$ is a sweep-out set for $\mu_{B}$ (i.e. $\left.\mu_{B}\left(\bigcup_{i \geqslant 1} \bar{\sigma}^{-i}[a]\right)=1\right)$.

We claim that $\int \phi d \mu_{B}>-\infty$. Set $C:=\sup _{n \geqslant 1} \operatorname{var}_{n+1} \phi_{n} \leqslant \sum_{k \geqslant 2} \operatorname{var}_{k} \phi$ and define $\bar{\phi}:=\sum_{i=0}^{\tau-1} \phi \circ \sigma^{i}$. Note that every $B \in \beta$ is a cylinder of length $n(B)+1$ where $n(B)$ is the unique value of $\tau$ on $B$. Now,

$$
\mathbb{E}_{\bar{\mu}}(\bar{\phi} \mid \beta)=\sum_{B \in \beta} 1_{B} \frac{1}{\bar{\mu}(B)} \int_{B} \bar{\phi} d \bar{\mu},
$$

so $\left\|\bar{\phi}-\mathbb{E}_{\bar{\mu}}(\bar{\phi} \mid \beta)\right\|_{\infty} \leqslant C$. Therefore,

$$
\begin{aligned}
\int \phi d \mu_{B} & =\mu[a] \int_{[a]} \bar{\phi} d \bar{\mu}_{B} \geqslant \mu[a] \int_{[a]} \mathbb{E}_{\bar{\mu}}(\bar{\phi} \mid \beta) d \bar{\mu}_{B}-C \\
& =\mu[a] \int_{[a]} \bar{\phi} d \bar{\mu}-C=\int_{\Sigma} \phi d \mu-C>-\infty .
\end{aligned}
$$

Consequently, $h_{\mu_{B}}(\sigma)+\int \phi d \mu_{B}$ is well-defined although it may be equal to $+\infty$. Therefore, by the variational principle, $h_{\mu_{B}}(\sigma) \leqslant-\int \phi d \mu_{B}<\infty$. Since $\mu_{B}$ is ergodic, Abramov's Formula applies and so $h_{\mu_{B}}(\sigma)=(1 / \mu[a]) h_{\bar{\mu}_{B}}(\bar{\sigma})=(1 / \mu[a]) H_{\bar{\mu}_{B}}(\beta)=$ $(1 / \mu[a]) H_{\bar{\mu}}(\beta)$. Therefore,

$$
H_{\bar{\mu}}(\beta)<\infty
$$


This implies that $\bar{\mu}$ satisfies the Rokhlin formula. Since $\mu$ is ergodic, the Kac and Abramov formulas both apply and so

$$
\begin{aligned}
\frac{1}{\mu[a]} h_{\mu}(\sigma) & =h_{\bar{\mu}}(\bar{\sigma})=-\int_{[a]} \log \frac{d \bar{\mu}}{d \bar{\mu} \circ \bar{\sigma}} d \bar{\mu}=-\frac{1}{\mu[a]} \int_{[a]} \log \frac{d \mu}{d \mu \circ \sigma^{\tau}} d \mu \\
& =-\frac{1}{\mu[a]} \int_{[a]} \sum_{i=0}^{\tau-1} \log \frac{d \mu}{d \mu \circ \sigma} \circ \sigma^{i} d \mu=-\frac{1}{\mu[a]} \int_{\Sigma} \log \frac{d \mu}{d \mu \circ \sigma} d \mu .
\end{aligned}
$$

Therefore, $\mu$ satisfies the Rokhlin formula as well. This shows that every ergodic equilibrium measure satisfies the Rokhlin formula.

Our next step is to prove that if $\mu$ is an ergodic equilibrium measure, then $d \mu / d \mu \circ \sigma=$ $g \bmod \mu \circ \sigma$ where $g$ is as in (1). Our argument is a variation on an argument of Ledrappier [17] (see also [28]) †.

We begin by showing that $\log g \in L^{1}(\mu)$ and that $\int(\varphi-\varphi \circ \sigma) d \mu=0$. Since $L_{\log g} 1 \leqslant 1, \log g \leqslant 0$ and so $\log g$ is one-sided integrable. We show that it is absolutely integrable. Set $g^{(n)}:=\prod_{i=0}^{n-1} g \circ \sigma^{i}, \bar{g}(x):=g^{(\tau(x))}(x)$. Since $\operatorname{var}_{1} \varphi<\infty$, $\varphi \cdot 1_{[a]} \in L^{1}(\bar{\mu})$ and $\int_{[a]}(\varphi-\varphi \circ \bar{\sigma}) d \bar{\mu}=0$. It follows that

$$
\begin{aligned}
\int \log g d \mu & =\mu([a]) \int_{[a]} \log \bar{g} d \bar{\mu}=\mu([a]) \int_{[a]}[\bar{\phi}-(\varphi-\varphi \circ \bar{\sigma})] d \bar{\mu} \\
& =\mu([a]) \int_{[a]} \bar{\phi} d \mu=\int \phi d \mu>-\infty .
\end{aligned}
$$

Therefore, $\log g \in L^{1}(\mu)$. It follows that $\varphi-\varphi \circ \sigma=\phi-\log g$ is absolutely integrable, because $\phi$ must be absolutely integrable $\left(\int \phi^{+}<\infty\right.$ as $\sup \phi<\infty$ and $\int \phi^{-}<\infty$, otherwise $\mu$ will not be an equilibrium measure). By the ergodic theorem,

$$
\int(\varphi-\varphi \circ \sigma) d \mu=\lim _{n \rightarrow \infty} \frac{1}{n}\left(\varphi-\varphi \circ \sigma^{n}\right)=\liminf _{n \rightarrow \infty} \frac{1}{n}\left(\varphi-\varphi \circ \sigma^{n}\right)=0
$$

so $\int(\varphi-\varphi \circ \sigma) d \mu=0$. Consequently, $h_{\mu}(\sigma)+\int \log g d \mu=0$.

Set $g_{\mu}:=d \mu / d \mu \circ \sigma$. We check that the transfer operator of $\mu$ is given by $\widehat{\sigma}_{\mu} f=$ $\sum_{\sigma y=x} g_{\mu}(y) f(y)$. The invariance of $\mu$ implies that $\sum_{\sigma y=x} g_{\mu}(y)=\widehat{\sigma}_{\mu} 1=1 \mu$-almost everywhere, so $g_{\mu}$ is a $g$-function. Since $\mu$ satisfies Rokhlin's Formula,

$$
\begin{aligned}
0 & =h_{\mu}(\sigma)+\int \log g d \mu=\int \log \frac{g}{g_{\mu}} d \mu \\
& =\int\left(\sum_{\sigma y=x} g_{\mu}(y) \log \frac{g(y)}{g_{\mu}(y)}\right) d \mu(x) \leqslant \int \log \left(\sum_{\sigma y=x, g_{\mu}(y)>0} g(y)\right) d \mu(x) \leqslant 0 .
\end{aligned}
$$

All inequalities must be equalities, so

$$
\sum_{\sigma y=x} g_{\mu}(y) \log \frac{g(y)}{g_{\mu}(y)}=\log \left(\sum_{\sigma y=x} g_{\mu}(y) \cdot \frac{g(y)}{g_{\mu}(y)}\right) \text { for } \mu \text {-almost all } x .
$$

By Lemma 2.4, for $\mu$-almost all $x$ there exists $c(x)$ such that

$$
y \in \sigma^{-1}\{x\}, \quad g_{\mu}(y)>0 \text { implies } g(y)=c(x) g_{\mu}(y) .
$$

$\dagger$ The arguments in $[\mathbf{1 7}, \mathbf{2 8}]$ only show that for almost every $x, d \mu / d \mu \circ \sigma(x)=g(x)$, but actually a stronger statement is needed: for almost every $x$, for all $y \in \sigma^{-1} x(d \mu / d \mu \circ \sigma(y)=g(y))$. 
Summing over $y \in \sigma^{-1}\{x\} \cap\left[g_{\mu}>0\right]$ gives $c(x)=\sum_{\sigma y=x, g_{\mu}(y)>0} g(y) \leqslant 1$. The last derivation shows that $\int \log c(x) d \mu(x)=0$ so $c(x)=1 \mu$-almost everywhere. For every $x$ such that $c(x)=1$ and $\sum_{\sigma y=x} g_{\mu}(y)=1, \sigma^{-1}\{x\} \subseteq\left[g_{\mu}>0\right]$, else $c(x)<\sum_{\sigma y=x} g(y) \leqslant 1$ (we have used the positivity of $g$ ). Therefore, for $\mu$-almost every $x$

$$
y \in \sigma^{-1}\{x\} \text { implies } g(y)=g_{\mu}(y) .
$$

This means that $L_{\log g_{\mu}}=L_{\log g}$ whence $L_{\log g}^{*} \mu=L_{\log g_{\mu}}^{*} \mu=\mu$. A calculation shows that $L_{\phi}^{*}\left(e^{-\varphi} \mu\right)=e^{-\varphi} \mu$ so (2) holds. By the discussion at the beginning of the proof, this proves the theorem.

Proof of Theorem 1.2. Assume without loss of generality that $P_{G}(\phi)=0$. By Lemma 2.3, $\phi=\log g+\varphi-\varphi \circ \sigma$ with $\varphi$ continuous and $g$ a sub $g$-function. The proof of Theorem 1.1 shows that if $\mu$ is an equilibrium measure, then (2) holds. Consequently, if $\nu:=e^{-\varphi} \mu$ and $h:=e^{\varphi}$, then

$$
L_{\phi}^{*} v=v \text { and } \int h d v=1
$$

For every $g \in L^{\infty}$,

$$
v\left(g L_{\phi} h\right)=v(g \circ \sigma \cdot h)=\mu(g \circ \sigma)=\mu(g)=v(g h)
$$

so $L_{\phi} h=h v$-almost everywhere. The identity $L_{\phi}^{*} v=v$ can be used to show that $v$ is finite and positive on cylinders and so since both $h$ and $L_{\phi} h$ are continuous, $L_{\phi} h=h$ everywhere.

\section{Equilibrium measures for piecewise expanding maps}

We prove Theorem 1.3. Let $(X, P, T)$ be a piecewise expanding map together with a piecewise Hölder-continuous potential $\phi: X \rightarrow \mathbb{R}$. We continue using the cylinder notation $\left[A_{0}, \ldots, A_{n}\right]:=\bigcap_{i=0}^{n} T^{-i} A_{i}$.

Fix some $T$-invariant probability measure $m$ on $X$. We prove that $P_{m}(\phi, T) \leqslant$ $P_{\text {top }}(\phi, T)$. We prove this under the extra assumption that $m$ is ergodic (otherwise use the ergodic decomposition).

The proof is based on a reduction to the symbolic dynamics of $T$, which we proceed to describe. Let $\operatorname{dom}\left(T^{n}\right) \subseteq X$ denote the domain of definition of $T^{n}$. The symbolic dynamics of $(X, P, T)$ is the left-shift $\sigma$ on:

$$
\begin{aligned}
& \Sigma(T)=\operatorname{Clos}\left(\left\{A=\left(A_{0}, A_{1}, \ldots\right) \in P^{\mathbb{N} \cup\{0\}}: \exists x \in X\right.\right. \\
& \text { such that for all } \left.\left.n \geq 0 x \in \operatorname{dom}\left(T^{n}\right) \text { and } T^{n} x \in A_{n}\right\}\right),
\end{aligned}
$$

where $\operatorname{Clos}(\cdot)$ denotes the closure in the compact space $P^{\mathbb{N} \cup\{0\}}$ (it is endowed with the product topology of the discrete topologies on $P$ ). Define $\pi: \Sigma(T) \rightarrow X$ by $\{\pi(A)\}=\bigcap_{n \geqslant 0} \overline{\left[A_{0} \ldots A_{n}\right]}$. As $T$ is piecewise expanding, $\pi$ is well defined. Moreover, if $\Delta:=\pi^{-1}(\partial P)$, then

$$
\pi: \Sigma(T) \backslash \bigcup_{k \geq 0} \sigma^{-k} \Delta \rightarrow X \backslash \bigcup_{k \geq 0} T^{-k} \partial P
$$


is bi-measurable, injective, surjective and satisfies $\pi \circ \sigma=T \circ \pi$. Next, define $\Phi: \Sigma(T) \rightarrow \mathbb{R}$ by $\Phi(A)=\lim _{n \rightarrow \infty} \inf \phi\left(\left[A_{0}, \ldots, A_{n}\right]\right)$ and note that

$$
\Phi(A)=\phi(\pi(A)) \quad \text { whenever } \quad \pi(A) \notin \partial P .
$$

It is easy to check that $\Phi$ is Hölder-continuous (respectively continuous) if $\phi$ is piecewise Hölder-continuous (respectively piecewise uniformly continuous); the distance on $\Sigma(T)$ is, as usual $d(A, B)=2^{-n}$, where $n$ is the smallest integer such that $A_{n} \neq B_{n}$ or $n=\infty$.

Set $S:=\bigcup_{k \geq 0} T^{-k} \partial P$. If $m(S)=0$ then $\pi:(\Sigma(T), \mathcal{B}(\Sigma(T)), m \circ \pi, \sigma) \rightarrow$ $(X, \mathcal{B}(X), m, T)$ is a measure-theoretic isomorphism, so $P_{m}(\phi, T)=P_{m \circ \pi^{-1}}(\Phi, \sigma)$. Also, $P_{m \circ \pi^{-1}}(\Phi, \sigma) \leqslant P_{\text {top }}(\Phi, \sigma)$, because of the variational principle for the topological pressure of a continuous function with respect to a homeomorphism of a compact metric space (see [27]). Therefore, $P_{m}(\phi, T) \leqslant P_{\text {top }}(\Phi, \sigma)$. It is routine to check that $P_{\text {top }}(\Phi, \sigma)=P_{\text {top }}(\phi, T)$ and it follows that $P_{m}(\phi, T) \leqslant P_{\text {top }}(\phi, T)$ whenever $m(S)=0$.

Suppose now that $m(S)>0$. We claim that $P_{m}(\phi, T)<P_{\text {top }}(\phi, T)$.

Indeed, $m(S)>0$ implies by invariance of $m$ that $m(\partial P)>0$. Thus our assumption that $P_{\text {top }}(\phi, \partial P, T)<P_{\text {top }}(\phi, T)$ and the following observation (the proof of which is given in $\$ 4)$ imply the claim.

PROPOSITION 3.1. Consider a piecewise expanding map $\tau$ together with a piecewise uniformly continuous potential $f$. Let $m$ be an ergodic invariant probability measure. If $m(S)>0$, then $P_{m}(f, \tau) \leqslant P_{\text {top }}(f, S, \tau)$.

This proves that $\sup _{m \in \mathcal{P}_{T}(X)} P_{m}(\phi, T) \leqslant P_{\text {top }}(\phi, T)$.

We now show that there exists $m$ such that $P_{m}(\phi, T)=P_{\text {top }}(\Phi, \sigma)$. Note that $\sigma: \Sigma(T) \rightarrow \Sigma(T)$ is expansive and that $\Phi$ is continuous (in fact, it is Höldercontinuous). It follows that there is a $\sigma$-invariant probability measure $\mu$ on $\Sigma(T)$ for which $P_{\mu}(\Phi, \sigma)=P_{\text {top }}(\Phi, T)\left[14\right.$, Theorem 20.2.10]. Set $m:=\mu \circ \pi^{-1}$. If $m(S)>0$ then $\mu\left(\pi^{-1} S\right)>0$ and so

$$
\begin{aligned}
P_{\text {top }}(\Phi, \sigma) & \equiv P_{\mu}(\Phi, \sigma) \leqslant P_{\text {top }}\left(\Phi, \pi^{-1} \partial P, \sigma\right) \quad(\text { Proposition 3.1) } \\
& \leqslant P_{\text {top }}(\phi, \partial P, T) \\
& <P_{\text {top }}(\phi, T) \quad(\text { by assumption }) \\
& =P_{\text {top }}(\Phi, \sigma)
\end{aligned}
$$

which is a contradiction. Therefore, $m(S)=0$. It follows that $\pi$ is an isomorphism between $(\Sigma(T), \mathcal{B}(\Sigma(T)), \mu, \sigma)$ and $(X, \mathcal{B}(X), m, T)$, and so $P_{m}(\phi, T)=P_{\mu}(\Phi, \sigma)=$ $P_{\text {top }}(\Phi, \sigma)$; we have already remarked that $P_{\text {top }}(\Phi, \sigma)=P_{\text {top }}(\phi, T)$. This proves Theorem 1.3(i).

We now turn to the finite multiplicity of the equilibrium measure, point (ii) of the theorem. We shall prove it by reduction to a Markov shift using the connected Markov diagram introduced in [5] by generalizing the construction of Hofbauer [13] for multidimensional dynamical systems. Let us recall its definition.

Definition 2. The (connected) Markov diagram is the directed graph $\mathcal{D}=(V, E)$ with vertices $V=\left\{T^{n} C: n \geqslant 0, C\right.$ is a connected component of $\left.Z \in P_{0}^{n}\right\}$ and edges 
$E=\{A \rightarrow B: \exists Z \in P$ such that $B$ is a connected component of $T(A) \cap Z\}$. This graph defines a one-sided Markov shift $(\Sigma, \sigma)$.

There is a natural projection onto the symbolic dynamics:

$$
q: \Sigma \rightarrow \Sigma(T)
$$

defined by $q(\alpha)=A$ such that $A_{n} \in P$ contains $\alpha_{n}$ for all $n \geq 0$. Obviously $q \circ \sigma=\sigma \circ q$ and $q$ is countable-to-one. We extend $\Phi$ to $\Sigma$ by setting $\Phi:=\Phi \circ q$ on $\Sigma$.

The proof of the second part of the theorem is based on constructing a certain type of isomorphism between the natural extensions of $\Sigma$ and $X$. We begin with some generalities. A weighted measurable dynamical system $(X, T, \phi)$ is a measurable map $T: X \rightarrow X$ together with a measurable function $\phi: X \rightarrow \mathbb{R}$ called the potential. A set $S \subseteq X$ is $P$-negligible if there exists $p<\sup _{\mu} P_{\mu}(\phi, T)$ such that for every ergodic invariant probability measure $\mu$, if $P_{\mu}(\phi, T)>p$ then $\mu(S)=0$.

Definition 3. Two weighted measurable dynamical systems $(X, T, \phi)$ and $(Y, S, \psi)$ are said to be $P$-isomorphic if there exist invariant subsets $X^{\prime} \subseteq X, Y^{\prime} \subseteq Y$ and a bimeasurable bijection $h: X^{\prime} \rightarrow Y^{\prime}$, such that $X \backslash X^{\prime}, Y \backslash Y^{\prime}$ are $P$-negligible, $h \circ T=S \circ h$ and $\phi=\psi \circ h$.

We will deduce part (ii) of the theorem from the following.

THEOREM 3.2. Let $(X, P, T)$ be a piecewise invertible map with a piecewise uniformly continuous potential $\phi: X \rightarrow \mathbb{R}$. If $P_{\mathrm{top}}(\phi, \partial P, T)<P_{\mathrm{top}}(\phi, T)$, then:

(1) the natural extensions $\dagger$ of $(X, T)$ and of $(\Sigma, \sigma)$ are $P$-isomorphic;

(2) $(\Sigma, \sigma)$ contains only finitely many maximal irreducible subchains (defined just after this) with pressure close to $P_{\text {top }}(\phi, T)$.

Recall that an irreducible part of an oriented graph is a subgraph with the property that any two vertices can be joined in both directions. A graph splits into its maximal irreducible parts (and a remaining part made of those vertices to which no path returns, but this part plays no role dynamically speaking). Recall also that the support of any ergodic invariant measure is contained in exactly one maximal irreducible subchain, i.e. the subchain defined by one of these maximal irreducible parts of the graph.

Before proving this theorem, let us see how it can be used to deduce part Theorem 1.3(ii). Note that $\left.\Phi\right|_{\Sigma}$ is bounded from above and is Hölder continuous, and therefore has finite Gurevich pressure since

$$
P_{G}\left(\left.\Phi\right|_{\Sigma}\right)=\sup _{\mu \in \mathcal{P}_{\sigma}(\Sigma)} P_{\mu}(\Phi, \sigma)=\sup _{\mu \in \mathcal{P}_{T}(X)} P_{\mu}(\phi, T)=P_{\text {top }}(\phi, T)<\infty .
$$

The second equality follows from Theorem 3.2. Under these conditions, Theorem 1.1 implies that each irreducible sub-shift has at most one equilibrium measure. Theorem 3.2(2) states that $\Sigma$ contains only finitely many maximal irreducible subchains with maximum pressure. Hence, $\Sigma$ has only finitely many ergodic equilibrium measures. By Theorem 3.2(1) this must then also be true for $T$, concluding the proof of Theorem1.3(ii).

$\dagger$ See the definition at the beginning of the proof 
Proof of Theorem 3.2. The strategy of the proof is the same as in [5] where the same statements were proved in the case $\Phi \equiv 0$ (entropy replacing pressure).

Let $p_{-}:\left(X_{-}, T_{-}\right) \rightarrow(X, T)$ be the natural extension,

$$
\begin{gathered}
X_{-}=\left\{x \in X^{\mathbb{Z}}: T\left(x_{n}\right)=x_{n+1} \forall n \in \mathbb{Z}\right\}, \\
T_{-}\left(\left(x_{n}\right)_{n \in \mathbb{Z}}\right)=\left(x_{n+1}\right)_{n \in \mathbb{Z}}, \text { and } \\
p_{-}\left(\left(x_{n}\right)_{n \in \mathbb{Z}}\right)=x_{0} .
\end{gathered}
$$

We also extend $\phi$ to $X_{-}$by setting $\phi:=\phi \circ p_{-}$. Define $\tilde{p}_{-}:\left(\Sigma_{-}, \sigma_{-}\right) \rightarrow(\Sigma, \sigma)$ and $\Phi$ on $\Sigma_{-}$similarly. Let $\Pi_{-}: \Sigma_{-} \rightarrow X_{-}$be the natural extension of $\Pi:=\pi \circ q: \Sigma \rightarrow X$ to a map from $\Sigma_{-}$to $X_{-}$.

It is well known that the projection $m \mapsto m \circ p_{-}$is a bijection between $\mathcal{P}_{T_{-}}\left(X_{-}\right)$and $\mathcal{P}_{T}(X)$ which preserves ergodicity, entropy and metric pressure.

The following notion plays a key role in the construction.

Definition 4. An invariant probability measure $\mu$ of $T_{-}$is said to be shadowed by the boundary if, for some integer $N$ and for $\mu$-almost every $x \in X_{-}$, there exist infinitely many positive integers $n$ such that $P_{0}^{n-1}\left(x_{-n}\right) \cap \Delta P \neq \varnothing$, where $\Delta P:=\bigcup_{k=0}^{N} T^{k}\left(\bigcup_{A \in P} \partial T A\right)$ and $P_{0}^{n-1}(x)$ is the element of $P_{0}^{n-1}$ containing $x$ if it exists or $\varnothing$.

Construct, as in [5], two invariant sets $X_{-}^{\prime} \subseteq X_{-}, \Sigma_{-}^{\prime} \subseteq \Sigma_{-}$with the following properties:

(1) The restriction, $\Pi_{-}: \Sigma_{-}^{\prime} \rightarrow X_{-}^{\prime}$ is a bi-measurable bijection and $\Pi_{-} \circ \sigma_{-}=$ $T_{-} \circ \Pi_{-}$.

(2) Let $\mu$ be an ergodic $T_{-}$-invariant probability on $X_{-}^{\prime}$. If $\mu\left(X_{-} \backslash X_{-}^{\prime}\right)=1$, then $\mu$ is shadowed by the boundary.

(3) Let $\mu$ be an ergodic $\sigma_{-}$-invariant measure on $\Sigma_{-}$. If $\mu\left(\Sigma_{-} \backslash \Sigma_{-}^{\prime}\right)=1$, then either $\mu \circ \Pi_{-}^{-1}$ is shadowed by the boundary, or $\mu\left(\tilde{p}_{-}^{-1} \Pi^{-1} \partial P\right)>0$.

For the construction of $X_{-}^{\prime}$ and $\Sigma_{-}^{\prime}$ and (1) see [5, Proposition 2.2]. Parts (2) and (3) are Propositions 2.6 and 2.7 there (observe that in contrast to Proposition 2.7 we work with the Markov shift defined by the Markov diagram and not the geometric Markov extension of [5]); to relate the two we have to code, hence a possible problem if $\left.\mu\left(\tilde{p}_{-}^{-1} \Pi^{-1} \partial P\right)>0\right)$.

To prove that the above restriction of $\Pi_{-}$is a $P$-isomorphism, we need to show that $X_{-} \backslash X_{-}^{\prime}$ and $\Sigma_{-} \backslash \Sigma_{-}^{\prime}$ are $P$-negligible.

We begin with the $P$-negligibility of $X_{-} \backslash X_{-}^{\prime}$. Property (1) says that every ergodic invariant probability measure $\mu$ carried by $X_{-} \backslash X^{\prime}$ must be shadowed by the boundary. The $P$-negligibility of $X_{-} \backslash X_{-}^{\prime}$ follows from the following proposition and our assumption that $P_{\text {top }}(\phi, \partial P, T)<P_{\text {top }}(\phi, T)$.

Proposition 3.3. Let $\mu$ be an invariant measure of $\left(X_{-}, T_{-}\right)$. If $\mu$ is shadowed by the boundary then $P_{\mu}\left(\Phi, T_{-}\right) \leqslant P_{\text {top }}(\phi, \partial P, T)$.

The proof of Proposition 3.3 is given in $\$ 4$.

Next, we prove the $P$-negligibility of $\Sigma_{-} \backslash \Sigma_{-}^{\prime}$. Consider some ergodic invariant probability $\mu$ measure carried by $\Sigma_{-} \backslash \Sigma_{-}^{\prime}$. It is enough to prove that $P_{\mu}\left(\Phi, \sigma_{-}\right) \leqslant$ $P_{\text {top }}(\phi, \partial P, T)$, because $P_{\text {top }}(\phi, \partial P, T)<P_{\text {top }}(\phi, T)=\sup _{m \in \mathcal{P}_{T}(X)} P_{m}(\phi, T) \leqslant$ $\sup _{m \in \mathcal{P}_{\sigma_{-}}\left(\Sigma_{-}\right)} P_{m}\left(\Phi, \sigma_{-}\right)$. 
Property (3) above says that either $\mu\left(\tilde{p}_{-}^{-1} \Pi^{-1} \partial P\right)>0$ or $\mu\left(\tilde{p}_{-}^{-1} \Pi^{-1} \partial P\right)=0$ and $\mu \circ \Pi_{-}^{-1}$ is shadowed by the boundary. In the first case, $v:=\mu \circ \tilde{p}_{-}^{-1} \circ q^{-1}$ is an ergodic $\sigma$-invariant measure on $\Sigma(T)$ which satisfies $\nu\left(\pi^{-1} \partial P\right)>0$. By Proposition 3.1, $P_{v}\left(\Phi,\left.\sigma\right|_{\Sigma(T)}\right) \leqslant P_{\text {top }}\left(\Phi, \pi^{-1} \partial P,\left.\sigma\right|_{\Sigma(T)}\right) \leqslant P_{\text {top }}(\phi, \partial P, T)$. Note that $m \mapsto m \circ\left(\tilde{p}_{-}\right)^{-1}$ and $m \mapsto m \circ q^{-1}$ both preserve entropy, because the first is a natural extension and the second is countable-to-one (see, e.g., Proposition 2.8 in [5]). Therefore, $P_{\mu}\left(\Phi, \sigma_{-}\right)=$ $P_{v}\left(\Phi,\left.\sigma\right|_{\Sigma(T)}\right) \leqslant P_{\text {top }}(\phi, \partial P, T)$.

In the second case, $\mu \circ \Pi_{-}^{-1}$ is shadowed by the boundary and Proposition 3.3 implies that $P_{\mu \circ \Pi_{-}^{-1}}\left(\Phi, T_{-}\right) \leqslant P_{\text {top }}(\Phi, \partial P, T)$. Since $\Pi_{-}$preserves entropy being a natural extension, $P_{\mu}\left(\Phi, \sigma_{-}\right)=P_{\mu \circ \Pi_{-}^{-1}}\left(\Phi, T_{-}\right)$and so again $P_{\mu}\left(\Phi, \sigma_{-}\right) \leqslant P_{\text {top }}(\phi, \partial P, T)$.

This proves that $\Sigma_{-} \backslash \Sigma_{-}^{\prime}$ is $P$-negligible and concludes the proof of part (1) of Theorem 3.2.

We now turn to part (2), which says that there are only finitely many subchains with large pressure. This is a strengthening of [5, Theorem C] even in the case of the zero potential, but under the additional assumption that $T$ is expanding. We obtain it by adapting Proposition 1.1 of [9].

Let $B(x, r) \subseteq X$ denote the ball of radius $r$ and center $x$ and define for $\alpha \in \Sigma$

$$
\epsilon(\alpha):=\sup \left\{r \geq 0: \exists n \geqslant 0 \text { such that } B\left(\Pi\left(\sigma^{n} \alpha\right), r\right) \subseteq \alpha_{n}\right\} .
$$

LEMmA 3.4. Let $\mathcal{V}$ be the set of vertices of the Markov diagram $\mathcal{D}$. For any $\delta_{0}>0$, there exists a finite subset $\mathcal{V}_{0} \subseteq \mathcal{V}$ such that for all $\alpha \in \Sigma \backslash \bigcup_{n \geq 0} \sigma^{-n} \Pi^{-1} \partial P$

$$
\text { if } \epsilon(\alpha)>\delta_{0}, \quad \text { then there exists } n \geq 0 \text { such that } \alpha_{n} \in \mathcal{V}_{0} \text {. }
$$

Proof. Let $k_{0}$ be an integer sufficiently large so that $\operatorname{diam} P_{0}^{k_{0}}<\frac{1}{2} \delta_{0}$. Define $\mathcal{V}_{0}$ to be the collection of the connected components $C$ of the subsets of the form

$$
T^{k_{0}} A \text { for some } A \in P_{0}^{k_{0}}
$$

which are such that $C$ contains a ball with radius $\delta_{0} / 2$. The finiteness of $\mathcal{V}_{0}$ follows from the fact that $T^{k_{0}}$ is piecewise uniformly continuous so that there is only a finite number of disjoint subsets $S \subseteq X$ such that $T^{k_{0}} S$ contains a ball with radius $\delta_{0}$.

Indeed, set $\mathcal{V}_{0}(A):=\mathcal{V}_{0} \cap\left\{C: C\right.$ is a connected component of $\left.T^{k} A\right\}$. By definition,

$$
\mathcal{V}_{0}=\bigcup_{\substack{A \in P_{0}^{k} \\ 0 \leqslant k \leqslant k_{0}}} \mathcal{V}_{0}(A)
$$

This is a finite union, because $P$ is finite. Therefore, it is enough to prove that $\mathcal{V}_{0}(A)$ is finite for every $A$. If this is not true, there are distinct $C_{1}, C_{2}, C_{3}, \ldots \in \mathcal{V}_{0}(A)$ with $C_{i} \supseteq B_{\delta_{0} / 2}\left(x_{i}\right)$. These balls are pairwise disjoint, because $C_{i}$ are pairwise disjoint (being connected components of the same set). Thus $\left\{x_{i}\right\}$ is a $\delta_{0} / 2$-separated sequence, which contradicts the compactness of $X$. This proves that $\mathcal{V}_{0}$ is finite.

Recall formula (2.1) of [5]. For $\alpha=\left(\alpha_{0}, \alpha_{1}, \ldots\right) \in \Sigma \backslash \bigcup_{n \geq 0} \sigma^{-n} \Pi^{-1} \partial P$,

$\alpha_{n+k}$ is the connected component of

$$
T^{k}\left(\alpha_{n} \cap\left[A_{n} \ldots A_{n+k}\right]\right) \text { which contains } \Pi\left(\sigma^{n+k} \alpha\right) .
$$


Let $\alpha \in \Sigma$ be as in the statement of the lemma with $\epsilon(\alpha)>\delta_{0}$. By definition, there is some integer $n \geqslant 0$ such that $\alpha_{n} \supseteq B\left(\Pi\left(\sigma^{n} \alpha\right), \delta_{0} / 2\right)$, so $\alpha_{n}$ contains a ball of radius $\delta_{0} / 2$. By the choice of $\delta_{0}, B\left(\Pi\left(\sigma^{n} \alpha\right), \delta_{0} / 2\right) \supseteq\left[A_{n} \ldots A_{n+k_{0}}\right]$ and so $\alpha_{n} \supseteq\left[A_{n} \ldots A_{n+k_{0}}\right]$. Therefore, by (3), $\alpha_{n+k_{0}}$ is also a connected component of $T^{k_{0}}\left[A_{n} \ldots A_{n+k_{0}}\right]$. This shows that $\alpha_{n+k_{0}} \in \mathcal{V}_{0}$.

LEMMA 3.5. For all $\epsilon>0$, there exists $\delta>0$ such that if $\mu$ is an invariant probability measure on $(\Sigma, \sigma)$ and $\epsilon(\alpha) \leqslant \delta$ for $\mu$-almost every $\alpha \in \Sigma$, then $P_{\mu}(\Phi, \sigma) \leqslant$ $P_{\text {top }}(\phi, \partial P, T)$.

We defer the proof of this lemma to the next section.

Proof of Theorem 1.3(ii). Fix $\epsilon \in\left(0, P_{\mathrm{top}}(\phi, T)-P_{\mathrm{top}}(\phi, \partial P, T)\right)$. Let $\delta>0$ be given by Lemma 3.5. Let $\mathcal{V}_{0}$ be the finite part defined by Lemma 3.4.

Claim. Any invariant probability measure $\mu$ of $\Sigma$ such that $\mu\left(\left\{\alpha \in \Sigma: \alpha_{0} \in \mathcal{V}_{0}\right\}\right)=0$ satisfies $P_{\mu}(\Phi, \sigma) \leqslant P_{\text {top }}(\phi, \partial P, T)+\epsilon$.

To prove this claim observe that by Lemma 3.4, $\mu$ must satisfy $\epsilon(\alpha) \leqslant \delta_{0}$ for $\mu$-almost every $\alpha$. But then we can apply Lemma 3.5 and get the claim.

It follows from the claim that any ergodic measure with pressure closer to $P_{\text {top }}(\phi, T)$ than this right-hand side must live on an irreducible subchain meeting $\mathcal{V}_{0}$, hence it must live on a finite number of maximal irreducible subchains as $\mathcal{V}_{0}$ is finite.

\section{Proof of the pressure estimates}

Proof of Proposition 3.1. $\mu$ is an invariant and ergodic probability measure of $\tau$ with $\mu(S)>0$. Fix $\epsilon>0$ arbitrarily small.

By the ergodic theorem, there exist $n_{1}<\infty$ and a subset $X_{1}$ of measure $>1-\frac{1}{2} \mu(S)$ such that for all $x \in X_{1}$ and all $n \geqslant n_{1}$

$$
\phi_{n}(x):=\sum_{k=0}^{n-1} \phi\left(\tau^{k} x\right) \geqslant n\left(\int \phi d \mu-\epsilon\right) .
$$

Note that $\mu\left(S \cap X_{1}\right)>\mu(S) / 2>0$.

Observe that

$$
\left|\phi_{n}(x)-\phi_{n}(y)\right|<\epsilon n
$$

for all $x, y$ in the same $n$-cylinder, for $n$ large enough. This is because of the piecewise uniform continuity of $\phi$ and because $\lim _{n \rightarrow \infty} \operatorname{diam} P^{n}=0$.

Consider $C_{n}$, the collection of $n$-cylinders meeting $S$. By definition,

$$
\sum_{A \in C_{n}} \sup _{x \in A} e^{\phi_{n}(x)} \leqslant C e^{n\left(P_{\mathrm{top}}(\phi, S, T)+\epsilon\right)}
$$

for some constant $C<\infty$ and all $n$. Remove from $C_{n}$ any cylinder that does not meet $X_{1}$. The resulting $C_{n}^{\prime}$ is a cover of $X_{1} \cap S$. By (5) and then (4),

$$
\sum_{A \in C_{n}} \sup _{x \in A} e^{\phi_{n}(x)} \geqslant \sum_{A \in C_{n}^{\prime}} \sup _{x \in A \cap X_{1}} e^{\phi_{n}(x)-\epsilon n} \geqslant \# C_{n}^{\prime} e^{n\left(\int \phi d \mu-3 \epsilon\right)} .
$$


We thus obtain

$$
\# C_{n}^{\prime} \leqslant C e^{\left(P_{\mathrm{top}}(\phi, S, \tau)-\int \phi d \mu+4 \epsilon\right) n} .
$$

Rudolph's formula for the entropy [21] states that if $\mu$ is an ergodic invariant probability measure and $P$ is a finite generator, then

$$
h_{\mu}(\tau)=\liminf _{n \rightarrow \infty} \frac{1}{n} \log R_{n}
$$

where $R_{n}$ is the minimum cardinality of a collection of $P$-cylinders with a union of measure of at least some fixed constant $0<\lambda<1$. Hence,

$$
h_{\mu}(\tau) \leqslant \liminf _{n \rightarrow \infty} \frac{1}{n} \log \# C_{n}^{\prime} .
$$

Hence we get, after taking the limits $n \rightarrow \infty$ and $\epsilon \rightarrow 0$,

$$
h_{\mu}(\tau) \leqslant P_{\text {top }}(\phi, S, \tau)-\int \phi d \mu
$$

from which the proposition follows immediately.

Proof of Proposition 3.3. Let $\mu_{-}$be an invariant and ergodic probability measure of $\left(X_{-}, T_{-}\right)$. Let $\mu$ be the corresponding measure on $(X, T)$. As is well known, $P_{\mu_{-}}\left(\Phi, T_{-}\right)=P_{\mu}(\Phi, T)$, so that it is enough to bound the latter quantity.

Pick $\epsilon>0$. Fix $n_{0}$ so large that for all $n \geqslant n_{0}$, the collection $S_{n}$ of $n$-cylinders, the closure of which meets $\partial P$, satisfies

$$
\sum_{A \in S_{n}} \sup _{x \in A} e^{\phi_{n}(x)} \leqslant e^{\left(P_{\mathrm{top}}(\phi, \partial P, T)+\epsilon\right) n} .
$$

Fix $n_{1}$ so large that for all $n \geqslant n_{1},\left|\phi_{n}(x)-\phi_{n}(y)\right|<\epsilon n$ for all $x, y$ in the same $n$-cylinder.

Let $n_{*}=\max \left(n_{0}, \epsilon^{-1} \log \left(\# P \cdot\|\phi\|_{\infty}\right) \cdot n_{1}\right)$. Increase $n_{*}$ if necessary so that the binomial coefficient $C_{n}^{\left[2 n / n_{*}\right]+1}$ is at most $e^{\epsilon n} /\left(n / n_{*}+1\right)$.

Indeed, by Stirling's Formula $n !=C^{ \pm 1}(n / e)^{n} n^{1 / 2}$, where $C^{ \pm 1}$ represents a function of $n$ with value in $\left(C^{-1}, C\right)$, where $C$ is independent of $n$. Hence, writing $\alpha:=2 / n_{*}$,

$$
\left(n / n_{*}+1\right) C_{n}^{\left[2 n / n_{*}\right]+1} \leqslant C^{3} \alpha n^{-1 / 2} \exp [(-\alpha \log \alpha-(1-\alpha) \log (1-\alpha)) n] \leqslant e^{\epsilon n}
$$

for $n$ large enough, if $n_{*}$ has been chosen large enough.

Define the measurable integer-valued function $n$ on $X_{-}$by

$$
n(x)=\min \left\{n \geqslant n_{*}: P^{n}\left(x_{-n}\right) \cap \Delta P \neq \varnothing\right\} .
$$

As $\mu_{-}$is shadowed by the boundary, this is well defined almost everywhere.

Let $N_{-}$be so large that $\mu_{-}\left(\left\{x \in X_{-}: n(x) \geqslant N_{-}\right\}\right)<\epsilon /\left(\# P \cdot\|\phi\|_{\infty}\right)$.

By the ergodic theorem, there exists $n_{3}<\infty$ and a measurable subset $X_{1} \subseteq X_{-}$such that for all $x \in X_{1}$ and $n \geqslant n_{3}$,

$$
\frac{1}{n} \#\left\{0 \leqslant k<n: n\left(T_{-}^{k} x\right) \geqslant N_{-}\right\}<\epsilon /\left(\# P \cdot\|\phi\|_{\infty}\right) .
$$

By the same reasoning as in the proof of Proposition 3.1, it is enough to prove that for all large $n$, the collection $C_{n}$ of $n$-cylinders that meet $X_{1}$ satisfies

$$
\sum_{A \in C_{n}} \sup _{x \in A} e^{\phi_{n}(x)} \leqslant C e^{n\left(P_{\mathrm{top}}(\phi, \partial P, T)+\epsilon\right)}
$$


Let $x \in X_{1}$ and $n \geqslant n_{*}$. We define intervals $\left[a_{i}, b_{i}\right)$ by induction. We set $a_{0}=n$. Provided $a_{i}$ is defined, we let $b_{i+1}=\max \left\{b \leqslant a_{i}: n\left(T_{-}^{b} x\right) \leqslant N_{-}\right\}$and $a_{i+1}=b_{i+1}-n\left(T_{-}^{b_{i+1}} x\right)$.

Let $i_{0}$ be the largest $i$ with $a_{i} \geqslant 0$. We claim:

(1) $\Delta P \cap P_{0}^{b_{i}-a_{i}}\left(p_{-} T_{-}^{a_{i}} x\right) \neq \varnothing$;

(2) $b_{i}-a_{i} \geqslant n_{*}$;

(3) $\#\left([0, n) \backslash \bigcup_{i=1}^{i_{0}}\left[a_{i}, b_{i}\right)\right) \leqslant\left(\epsilon / \log \left(\# P \cdot\|\phi\|_{\infty}\right)\right) n$.

Therefore, the itinerary $A_{0} A_{1} \ldots A_{n-1} \in P^{n}$ (or more precisely in $\left(p_{-}^{-1} P\right)^{n}$ ) determining the $n$-cylinder containing $x$ can be described completely by specifying:

(1) an integer $0 \leqslant r \leqslant n / n_{*}$ giving the number of intervals $\left[a_{i}, b_{i}\right)$;

(2) the starting and ending positions of each one of these intervals;

(3) the symbols $A_{a_{i}} A_{a_{i}+1} \ldots A_{b_{i}-1}$ picked among the itineraries of the same length of points in $\Delta P$;

(4) the symbols at the remaining places.

Hence, writing $n^{\prime}=\sum_{i=1}^{i_{0}}\left(b_{i}-a_{i}\right)$,

$$
\begin{aligned}
\sum_{A \in C_{n}} \sup _{x \in A} e^{\phi_{n}(x)} \leqslant & \sum_{r=0}^{\left[n / n_{*}\right]} \sum_{0 \leqslant a_{r}<b_{r}<\cdots<a_{1}<b_{1}<n} \prod_{i=1}^{r} \sum_{B \in P_{0}^{b_{i}-a_{i}-1} \mid \bar{B} \cap \Delta P \neq \varnothing} \\
& \times \sup _{y \in B} e^{\phi_{b_{i}-a_{i}}(y)}\left(\# P \cdot e^{\left.\|\phi\|_{\infty}\right)^{n-n^{\prime}}}\right. \\
\leqslant & \left(n / n_{*}+1\right) C_{n}^{2\left[n / n_{*}\right]} e^{n\left(P_{\mathrm{top}}(\phi, \partial P, T)+2 \epsilon\right)} e^{\epsilon n} \\
\leqslant & e^{n\left(P_{\mathrm{top}}(\phi, \partial P, T)+4 \epsilon\right)},
\end{aligned}
$$

proving the claim.

Proof of Lemma 3.5. Let $\mu$ be an invariant probability measure on $(\Sigma, \sigma)$ such that for almost every $\alpha, \epsilon(\alpha)<\delta$. Fix $D_{0}$ such that $\mu\left(\left\{\alpha: \alpha_{0}=D_{0}\right\}\right)>0$. The proof of Proposition 1.1 in [9] shows that if $\delta>0$ is small enough then for every $n \geq 0$ there is a collection $C_{n}$ of $n$-cylinders containing all the points $\alpha \in[D]$ that satisfy $\epsilon(\alpha)<\delta$ such that

$$
\sum_{A \in C_{n}} \sup _{A} e^{\Phi_{n}(x)} \leqslant C e^{\left(P_{\mathrm{top}}(\phi, \partial P, T)+\epsilon\right) n} .
$$

The union of the cylinders in $C_{n}$ covers $D_{0}$ modulo $\mu$, thus its $\mu$-measure is positively lower-bounded. However, we have seen (see the proof of Proposition 3.1) that this implies that $P_{\mu}(\Phi, \sigma) \leqslant P_{\text {top }}(\phi, \partial P, T)+\epsilon$.

Acknowledgements. The second author stayed at the IHES while this work was done and would like to thank the institute and its staff for their hospitality and support.

\section{REFERENCES}

[1] J. Aaronson. An Introduction to Infinite Ergodic Theory (Mathematical Surveys and Monographs, 50). American Mathematical Society, Providence, RI, 1997.

[2] R. Bowen. Equilibrium States and the Ergodic Theory of Anosov Diffeomorphisms (Lecture Notes in Mathematics, 470). Springer, Berlin, 1975. 
[3] J. Buzzi. Intrinsic ergodicity for smooth interval maps. Israel J. Math. 100 (1997), 125-161.

[4] J. Buzzi. Intrinsic ergodicity of affine maps in $[0,1]^{d}$. Monat. Math. 124 (1997), 97-118.

[5] J. Buzzi. Markov extensions for multidimensional dynamical systems. Israel J. Math. 112 (1999), 357-380.

[6] J. Buzzi. Thermodynamical formalism for piecewise invertible maps: absolutely continuous invariant measures as equilibrium states. Smooth Ergodic Theory and its Applications (Seattle, WA, 1999) (Proc. Sympos. Pure Math., 69). American Mathematical Society, Providence, RI, 2001, pp. 749-783.

[7] J. Buzzi. On entropy-expanding maps. Preprint, 2000.

[8] J. Buzzi, F. Paccaut and B. Schmitt. Conformal measures for multi-dimensional piecewise invertible maps. Ergod. Th. \& Dynam. Sys. 21 (2001), 1035-1049.

[9] J. Buzzi and V. Maume-Deschamps. Decay of correlations for piecewise invertible maps in higher dimensions. Israel J. Math. 131 (2002), 203-220.

[10] B.M. Gurevich. Topological entropy of a countable Markov chain. Dokl. Akad. Nauk SSSR 187 (1969), 715-718.

[11] B.M. Gurevich. Shift entropy and Markov measures in the space of paths of a countable graph. Dokl. Akad. Nauk SSSR 192 (1970), 963-965.

[12] B.M. Gurevich and S.V. Savchenko. Thermodynamic formalism for countable symbolic Markov chains. Uspekhi Mat. Nauk. 53(2) (1998), 3-106. (Engl. transl. Russian Math. Surv. 53(2) (1998), 245-344.)

[13] F. Hofbauer. Piecewise invertible dynamical systems. Probab. Th. Rel. Fields 72 (1986), 359-386.

[14] A. Katok and B. Hasselblatt. Introduction to the Modern Theory of Dynamical Systems (Encyclopedia of Mathematics and its Applications, 54). Cambridge University Press, Cambridge, 1995.

[15] M. Keane. Strongly mixing g-measures. Invent. Math. 16 (1972), 309-324.

[16] B.P. Kitchens. Symbolic Dynamics: One-sided, Two-sided and Countable State Markov Shifts. Springer (Universitext), 1998.

[17] F. Ledrappier. Principe variationnel et systèmes dynamiques symboliques. Z. Wahrsch. Gebiete 30 (1974), 185-202.

[18] R. D. Mauldin and M. Urbański. Gibbs states on the symbolic space over an infinite alphabet. Israel J. Math. 125 (2001), 93-130.

[19] W. Parry. Intrinsic Markov Chains. Trans. Amer. Math. Soc. 112 (1964), 55-66.

[20] D. Ruelle. A measure associated with Axiom A attractors. Amer. J. Math. 98(3) (1976), 619-654.

[21] D. Rudolph. Fundamentals of Measurable Dynamics. Oxford Press, 1990.

[22] O. Sarig. Thermodynamics formalism for countable Markov shifts. Tel-Aviv University Dissertation, 2000 .

[23] O. Sarig. Thermodynamic formalism for countable Markov shifts. Ergod. Th. \& Dynam. Sys. 19 (1999), $1565-1593$.

[24] O. Sarig. Thermodynamics formalism for null recurrent potentials. Israel J. Math. 121 (2001), $285-311$.

[25] O. Sarig. Phase transitions for countable Markov shifts. Commun. Math. Phys. 217 (2001), 555-577.

[26] O. Sarig. Existence of Gibbs measures for countable Markov shifts. Proc. Amer. Math. Soc. 131 (2003), 1751-1758.

[27] P. Walters. An Introduction to Ergodic Theory (Graduate Texts in Mathematics, 79). Springer, New YorkBerlin, 1982.

[28] P. Walters. Ruelle's operator theorem and g-measures. Trans. Amer. Math. Soc. 214 (1978), 375-387. 